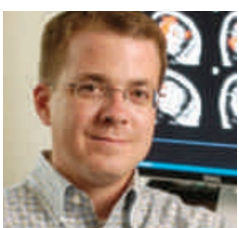

\title{
Brain scans need a rethink
}

\section{Head movement can bias brain imaging results, undermining a leading theory on the cause of autism, say Ben Deen and Kevin Pelphrey.}

Kevin Pelphrey

$\mathrm{O}$ ne of the most popular and widely accepted theories on the cause of autism spectrum disorders attributes the condition to disrupted connectivity between different regions of the brain. This 'connectivity hypothesis' claims that the social and cognitive abnormalities in people with autism can be explained by a dearth of connections between distant regions of the brain ${ }^{1}$. Some flavours of this theory also predict more connections between nearby brain regions.

Recent studies, however, have found that when a person moves their head while undergoing functional magnetic resonance imaging (fMRI) - a method that maps how different neuroanatomical structures of the brain interact in real time, its functional connectivity - it looks like the neural activity observed in autism. That's a sobering discovery: it means that a major source of evidence for a leading hypothesis on autism, and one that several research teams have pursued for years, may arise from an artefact.

Many studies have investigated functional connectivity in the brains of people with autism, and most have reported evidence supporting the connectivity hypothesis. These findings are consistent with results from some animal models of autism, and from studies using diffusion tensor imaging, which measures the bundles of fibres connecting parts of the brain.

But three studies published in 2012

have come to the same conclusion: head motion leads to systematic biases in fMRI-based analyses of functional connectivity ${ }^{2-4}$. Specifically, motion makes it appear as if long-range connections are weaker than they really are, and that short-range connections are stronger than they really are.

This bias affects all functional connectivity analyses, but it is particularly insidious for studies of autism. That's because it would lead to precisely the patterns that have been observed in fMRI scans of children with autism, and because children with autism typically move more than unaffected children do.

How can autism researchers overcome this bias? One approach would be to define a measure of head motion in each participant over the course of a scan - for instance, to compute the displacement of the head between consecutive time points, and average this over time. Researchers can then check that the autism and control groups are well matched on this measure, or include this value as a nuisance variable in regression analyses.

The matching, however, would need to be precise: as one of the new studies showed, even a difference as small as 0.004 millimetre in average head motion across groups of patients can lead to significant differences in correlation strengths ${ }^{4}$.

Furthermore, it is likely that motion artefacts can persist even when groups are matched on mean head motion. First, there is some evidence that head motion relates to functional connectivity measures in a nonlinear fashion ${ }^{2,4}$. If that's true, it would not be sufficient to account for only linear effects of motion. Even if head motion doesn't significantly differ across groups, a nonlinear function of head motion could.
Second, a given estimate of average motion can correspond to rather different scenarios - a few isolated but large movements, or constant small movements - which would have different effects on fMRI signals and on measures of functional connectivity. For instance, it has been shown that large, jerky movements lead to brief spikes in fMRI signal strength.

Based on this observation, cognitive neuroscientist Steven Petersen and his colleagues at Washington University in St Louis, Missouri, propose a strategy for mitigating these head-motion artefacts ${ }^{2}$. They recommend removing periods of high motion. They have shown that this technique, which they call 'scrubbing', corrects at least some of the spurious correlations caused by head motion.

This approach is promising but many questions remain. For instance, the extent of motion-induced correlations even after scrubbing is not well understood. Moreover, the optimal motion threshold for removing periods has not been studied in detail. Still, performing scrubbing in addition to group matching on average motion estimates and other standard noise-reduction methods represents current best practice in functional connectivity research.

So far, only a few research groups have used scrubbing or similar techniques in fMRI studies on autism ${ }^{5,6}$. Given the importance of understanding whether previous results on functional connectivity in autism are real or merely motion-related artefacts, we hope that the authors of fMRI functional connectivity studies in autism (and particularly those reporting results consistent with the connectivity hypothesis) will reanalyse their data using these techniques.

Scrubbing can be easily implemented using freely available software such as Artifact Detection Tools or by implementing custom modifications to the programs. Reanalysing data should be facilitated by tools provided by the US National Database for Autism Research, part of the National Institutes of Health. In addition, a trove of information has recently become publicly available in the Autism Brain Imaging Data Exchange - a collection of resting-state fMRI imaging data sets from 539 individuals with autism and 573 controls. Revisiting fMRI studies with these approaches would help establish whether there really is a connectivity deficit in the brains of people with autism.

Ben Deen is a graduate student in brain and cognitive sciences at the Massachusetts Institute of Technology in Cambridge. Kevin Pelphrey is the Harris Associate Professor in the Child Study Center at Yale University in New Haven, Connecticut.

email:kevin.pelphrey@yale.edu

1. Just, M. A. et al. Brain 127, 1811-1821 (2004).

2. Power, J. D. et al. Neuroimage 59, 2142-2154 (2012).

3. Satterthwaite, T. D. et al. Neuroimage 60, 623-632 (2012).

4. Van Dijk, K. R. A. et al. Neuroimage 59, 431-438 (2012).

5. Kennedy, D. P. et al. Neuroimage 39, 1877-1885 (2008).

6. Deen, B. et al. International Meeting for Autism Research (Philadelphia, 2010). 